\title{
INFLUENCE OF DRY CLEANING ON THE ELECTRICAL RESISTANCE OF SCREEN PRINTED CONDUCTORS ON TEXTILES
}

\author{
Ilda Kazani ${ }^{1,2}$, Gilbert De Mey³, R. Klepacko,4, Carla Hertleer ${ }^{1}$, Genti Guxho², Lieva Van Langenhove ${ }^{1}$, Andrzej Napieralski ${ }^{4}$ \\ 1Department of Textiles Ghent University, Technologiepark 907, 9052 Zwijnaarde, Belgium \\ Carla.Hertleer@UGent.be; Lieva.Vanlangenhove@UGent.be \\ 2Polytechnic University of Tirana, Department of Textile and Fashion, Square 'Mother Teresa', No.4, Tirana, Albania \\ ikazani@fim.edu.al; gguxho@fim.edu.al \\ ${ }^{3}$ Department of Electronics and Information Systems, Ghent University, Sint Pietersnieuwstraat 41, 9000 Ghent, Belgium \\ Gilbert.DeMey@elis.UGent.be \\ ${ }^{4}$ Department of Microelectronics and Computer Science, Technical University of Lodz, 221/223 Wolczanska St., Lodz, Poland \\ r.klepaczko@gmail.com; napier@p.lodz.pl
}

\begin{abstract}
:
Electrically conducting inks were screen printed on various textile substrates. The samples were dry cleaned with the usual chemicals in order to investigate the influence of the mechanical treatment on the electrical conductivity. It was found that dry cleaning has a tremendous influence on this electrical conductivity. For several samples, it is observed that the electrical resistance increases with the square of the number of dry cleaning cycles. In order to explain this observation a theoretical model and a numerical simulation have been carried out, by assuming that dry cleaning cycles introduce a crack in the conducting layer. The theoretical analysis and the numerical analysis both confirmed the experimental observations.
\end{abstract}

\section{Keywords:}

Screen-printed textile conductors, conductive ink, textile, dry cleaning, electrical resistance

\section{Introduction}

Screen printing with conductive ink is a technique mainly used in the field of electronics. However, over the last decade different groups have screen printed with conductive silver-based inks on different woven and nonwoven fabrics for applications such as electrodes for monitoring vital signs, interconnections in circuits, heating elements or planar antennas for wireless off-body communication [1-9]. However, when integrated in wearable textile systems, consumers will only accept them if they do not require any special maintenance preferably without removing all devices. Some of these wearable textile systems require regular washing processes and some others dry cleaning process. Karaguzel et al. [6] have worked on laundering printed electroconductive textiles in a washing machine where they have concluded that the printed electroconductive textiles resulted in a loss of conductivity. Kazani et al. [10] worked on preserving the electroconductive properties after 60 dry cleaning cycles of screen-printed electroconductive flexible substrates. Both of these works concluded that the maintenance procedure, laundering or dry cleaning, of the printed electroconductive substrates had an influence on their resistance.

The influence of washing on electric charge decay of fabrics with conductive yarns was studied in [11] and the influence of washing on the electrostatic properties of polyester woven fabrics containing S-Shield conductive yarns was studied in [12]. In both papers the vertical electrical resistance and surface resistivity were measured, for knitted and woven fabrics with conductive yarns inserted at specified intervals, before and after five washing cycles. It was found that the values of the vertical electrical resistance and surface resistivity of these fabrics increase very distinctly after five washing cycles.

This paper reports on the influence of dry cleaning on the electrical resistance of screen-printed conductors on textile along with a theoretical model and simulations to explain the observed phenomena. For the experimental part of this paper, electroconductive layers were screen-printed onto two flexible substrates.

From our investigation it was seen that the dry cleaning process has a tremendous influence on the electrical conductivity. Here it is confirmed with theoretical and numerical analysis, which was also observed during the experiments, that the electrical resistance increases with the square of the number of dry cleaning cycles.

\section{Experimental results}

Silver-based electroconductive inks were screen printed on two different substrate materials: a flexible polyurethane foam (Urecom) and a polyester nonwoven (PES). The screen-printed area was a square of 6 by $6 \mathrm{~cm}$. To measure the electrical resistance of this square, the Van Der Pauw method was applied [13-15]. This technique uses four contacts put on the boundary of the conducting layer.

It has the particular advantage that the parasitic contact resistance does not influence the measurements. A known electrical current $I$ is fed through two contacts (e.g. contacts 1 
and 2 in Figure 1) and the resulting voltage drop $\mathrm{V}$ (in contacts 3 and 4 of Figure 1) across the two other contacts is measured. We define in this paper the value $\mathrm{R}=\mathrm{V} / \mathrm{l}$ as the resistance of the sample, although a resistance is normally defined if only two contacts are involved. Anyway, the value $R$ is always proportional to the resistivity $\rho$ of the sample. In order to calculate this resistivity $\rho$ from the obtained $R$ value one needs to solve a potential problem that takes the geometry of the sample into account. This job is not always that obvious and will be omitted here [14]. As we are only interested in ageing properties and taking into account that $R$ is always proportional to $\rho$, we will continue with the resistance value $R$ without any geometry correction.

In a first series of experiments four different silver-based electroconductive inks were deposited on the Urecom and on the PES substrates. The four inks are labelled as ink1, ink2, ink3 and ink4. These inks have been supplied by DuPont (ink 1), Acheson (ink 2) and Sun Chemical (ink 3 and 4).

After the prior measurement of the resistance, all samples were dry cleaned up to maximum 60 times; after $N=5,10,15, \ldots 60$ dry cleaning cycles, the resistance was measured again to observe the ageing behaviour. The dry cleaning procedure was carried out with the usual chemical cleaning agent. But dry cleaning involves also a lot of mechanical movements of the samples. We believe that this mechanical treatment is mainly responsible for ageing of the electric conducting layers.

The experimental results are shown in Figure 2.

On the vertical axis, the values $R-R_{0}$ were plotted. $R_{0}$ is the resistance value at the initial stage $(N=0)$, and $R$ is the resistance after $\mathrm{N}$ dry cleaning cycles. The reason for showing $R-R_{0}$, or the change in resistance, is to allow a better comparison with the theoretical and numerical analysis as will be explained further on in the next sections. The horizontal axis shows the number of dry cleaning cycles N. A double logarithmic plot is used to allow a better comparison with the theoretical analysis and the numerical simulations.

First of all one notices that the resistance values increase a lot due to the dry cleaning process. Typically the resistance value increases 10 to 50 times for $N=60$ cycles. If these conductors have to supply an electrical current to any kind of wearable electronic devices, the severe increase of the resistance has to be taken into account during the design phase.

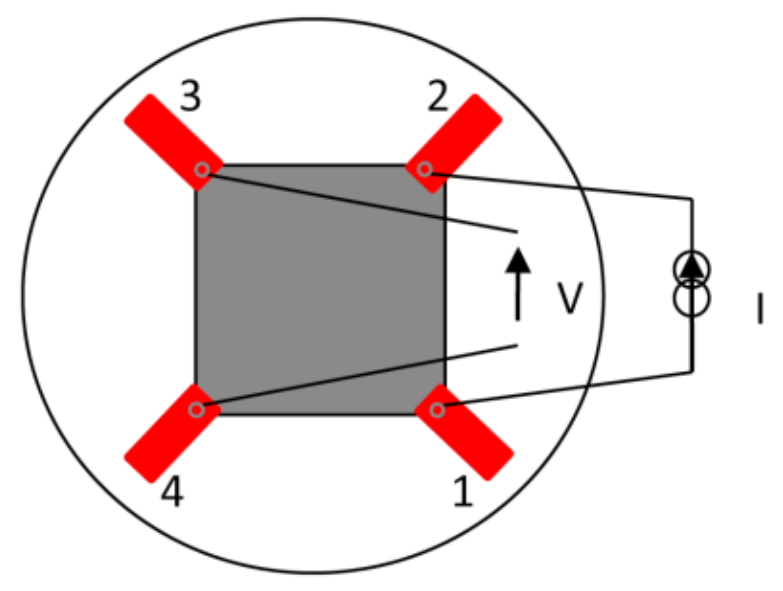

Figure 1. The Van Der Pauw set-up to measure electrical resistance [10]

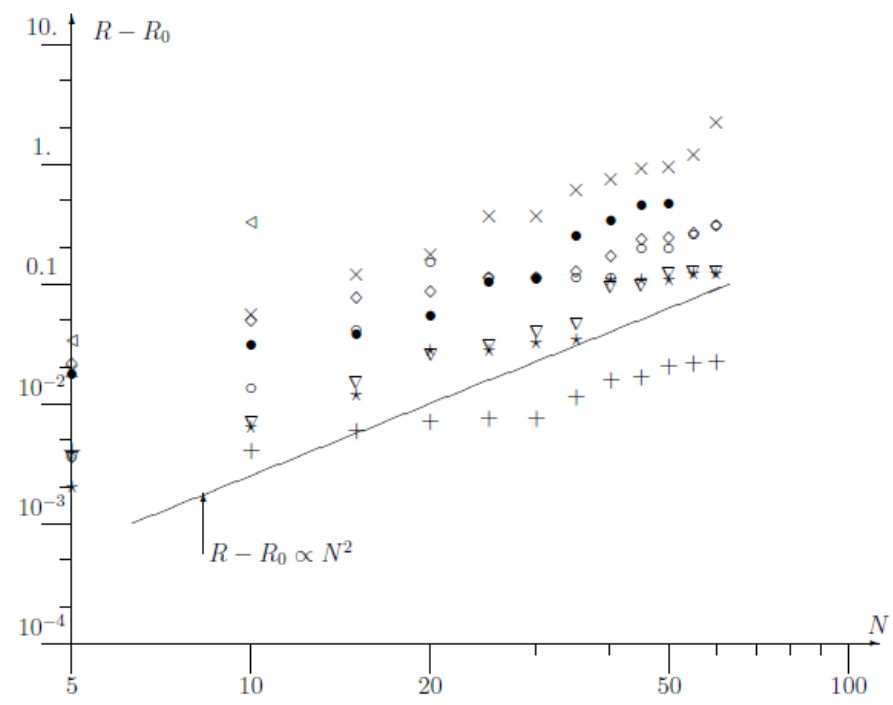

Figure 2. Ageing behaviour of the electric resistance of different inks on Urecom substrates [ink1 $(\bullet)$, ink2 $(\times)$, ink3 (०) and ink4 $(\nabla)$ ] and PES substrates [ink1 $(\triangleleft)$, ink2 $(+)$, ink3 $(\diamond)$ and ink4 $(\star)]$. 
In Table 1, the values of the initial resistances (i.e. any dry cleaning process or $\mathrm{N}=0$ ) are listed.

Table 1. Initial resistance values

\begin{tabular}{|c|c|c|}
\hline $\mathbf{R}_{\mathbf{0}}(\boldsymbol{\Omega})$ & Urecom & PES \\
\hline Ink 1 & $0.0070(\bullet)$ & $0.0140(\triangleleft)$ \\
\hline Ink 2 & $0.0105(\times)$ & $0.0025(+)$ \\
\hline ink 3 & $0.0040(\circ)$ & $0.0100(\diamond)$ \\
\hline ink 4 & $0.0020(\nabla)$ & $0.0005(\star)$ \\
\hline
\end{tabular}

The wide scattering of the numerical values is due to the material properties of both the inks and the substrates. The inks contain organic solvents, which are differently absorbed in Urecom and PES. This aspect has been discussed in detail in a previously published paper [10]. The influence of the substrate material is not easy to describe due to some contradictory results. For ink1 and ink3, the Urecom gives rise to a lower resistance, whereas the opposite result is found for ink2 and ink4 although ink3 and ink4 are from the same supplier. From the ageing point of view (Figure 2), one observes roughly the same trend for all samples: the resistance increases with the number of dry-cleaning cycles. One exception was found for ink1 on a PES substrate where no electric conduction was possible for $N>10$.

The most obvious explanation is that the dry cleaning creates a lot of mechanical bendings of the samples. Due to the flexibility of the textile substrates, these bendings will create microcracks in the conducting layers. This phenomenon was also confirmed by the inspection of the dry cleaned layers under a stereo microscope [10]. Any crack in an electric conductor gives an inevitable rise to the electric resistance, because the current paths are partially blocked.

From Figure 2, it is clear that several samples follow the slope of the trendline. For the Urecom substrate it happens for all the four inks. Concerning the PES substrate only the sample with ink 4 follows the trendline. The samples with ink2 (+) and ink3 $(\diamond)$ show a lower slope. The PES substrate with ink1 $(\triangleleft)$, however, has a totally different behaviour. After 10 dry cleaning cycles, the resistance has increased from $R_{0}=0.0140 \Omega$ to $R=0.3428 \Omega$ and after 15 cycles, no more electrical conduction was observed.

In a second experiment just one ink (ink1) was screen printed on the Urecom and on the PES substrate. The ageing results are shown in Figure 3.

For the foam substrate the initial resistance was $R_{0}=0.009$ $\Omega$ and $R_{0}=0.024 \Omega$ for the nonwoven PES. A trendline ( has also been drawn. This time the experimental results do not follow the slope of the trendline. For the foam substrate the resistance varied from $R=0.009 \Omega$ to $R=0.022 \Omega$ after 60 cry cleaning cycles. For the nonwoven PES substrate one observed $R_{0}=0.024 \Omega$ and $R=0.158 \Omega$. This time the change of resistance is no more than 5 . A possible explanation for this phenomenon is that these substrates are less flexible so that the mechanical movements during dry cleaning have less influence on the ageing of the conducting layers. Just like in the first set of experiments, the influence of the substrate on the absolute value of the resistance is considerable.

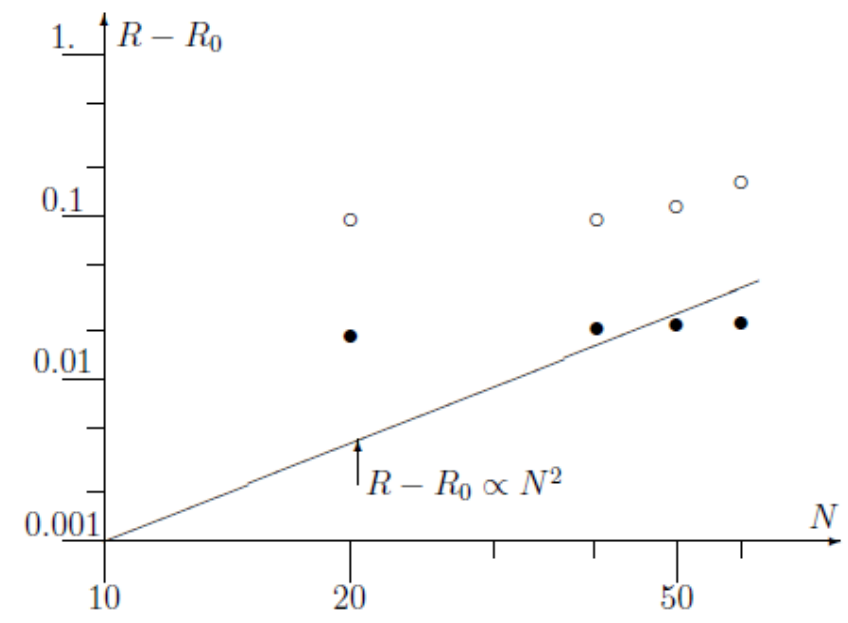

Figure 3. Ageing behaviour of the electric resistance of ink1 on Urecom $(\bullet)$ and nonwoven PES substrate $\left({ }^{\circ}\right)$

\section{Modelling}

Setting up a mathematical model for washing cycles is far from being obvious. In this contribution, a simple theoretical approach is presented.

First of all we assume that a linear shaped crack exist in the conducting layer. During each washing cycle, it is assumed this crack grows a certain amount. It is then sufficient to find out the change of the electric resistance as a function of the crack length. This analysis gives rise to an analytical calculation as will be outlined further on in the next section. It will be found that the change of the total resistance is proportional to the square of the crack length and hence with the square of the number of dry cleaning cycles.

Second, a numerical analysis is presented. The conducting layer is represented by a two dimensional array of resistors aligned along a rectangular Cartesian system. A washing cycle is simulated by the removal of one or more resistors from this electric network, which can be interpreted as a microcrack. The choice of a resistor for the next removal is done with a random generator so that this time we are not dealing with a single growing crack but with many cracks distributed all over the conducting layer. This numerical procedure is repeated until electrical conduction turns out to be no longer possible. As long as the number of removed resistors is not too big, it is found that the change of the total resistance is proportional to the square of the number of dry cleaning cycles.

It will be proved theoretically in the next section that for a strip with one crack the change of resistance is proportional to the square of the crack length. One assumes that the crack length grows proportionally with the number of dry cleaning cycles it can be stated that the change of the resistance will be proportional to the square of the number of dry cleaning cycles. With the numerical simulations a crack was represented by removing a single 
resistor from a large network of interconnected resistors. Again it was found that the overall resistance increased proportional to the square of the number of removed resistors. It is reasonable to assume that the number of cracks is proportional to the number of dry cleaning cycles. Hence one ends up with the relation $R-R_{0} \propto N^{2}$. Hence this relation, which is a straight line in a double logarithmic plot, has also been plotted in Figure 1. As will be made clear later on in this article, only the slope of this trendline should be taken into account.

\subsection{Theoretical analysis}

Let us first consider an electric conducing path with a width a as shown in Figure 4 by (a).

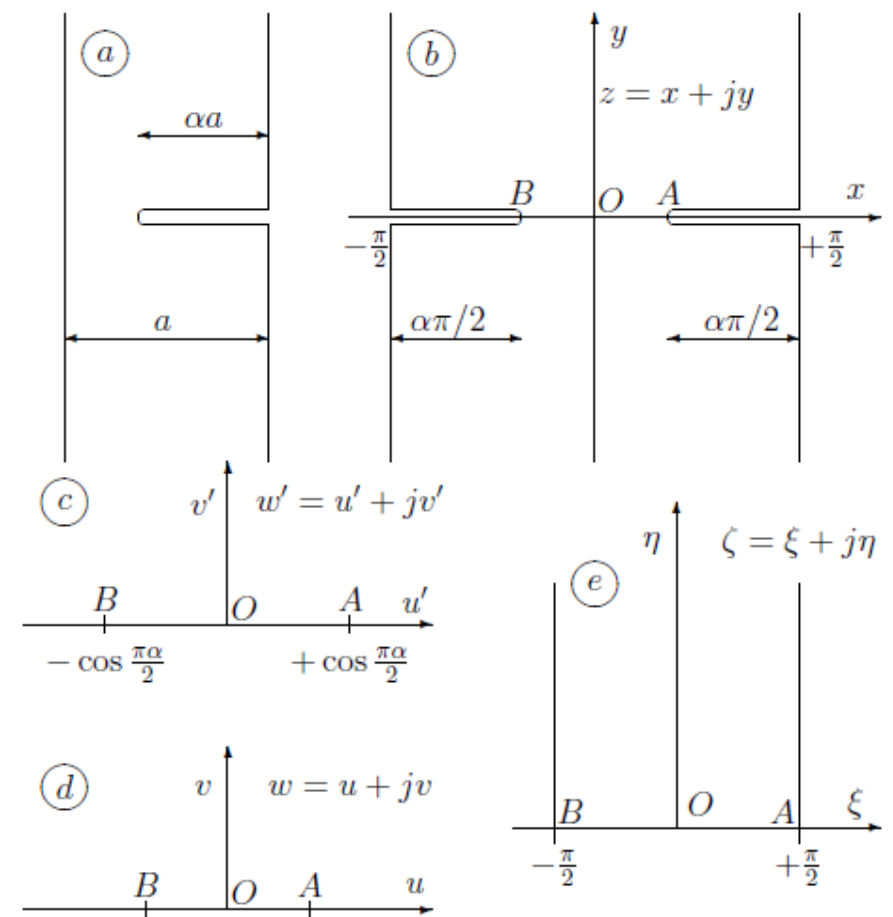

Figure 4. Track (width a) with a crack aa and the related conformal mappings to obtain the potential distribution

A crack with a length $\alpha a(0<\alpha a)$ has also been drawn. To simplify the calculations it is assumed the crack is directed along the $x$-axis. Moreover, only one crack is taken into consideration here. The potential problem to be solved can be easily performed analytically using conformal mapping techniques as will explained further on [16-17].

The first step in the calculation is to mirror the geometry of Figure $4 \mathrm{a}$ giving us the band structure shown in Figure $4 \mathrm{~b}$.

For mathematical reasons only the upper half plane (i.e. $y>0$ ) will be considered further on. Due to symmetry, this has no influence on the analysis. The track of Figure $4 b$ has a width $2 a$ and two cracks placed symmetrically with respect to the $y$-axis. With the conformal transformations only the upper half planes will be transformed.

The geometry of Figure $4 \mathrm{~b}$ will be mapped onto the upper half plane $v^{\prime}>0$ of Figure $4 \mathrm{c}$ by: $\omega^{\prime}=u^{\prime}+j v^{\prime}=\sin \left(\frac{\pi z}{2 a}\right)=\sin \left(\frac{\pi z}{2 a}\right) \cosh \left(\frac{\pi z}{2 a}\right)+j \cos \left(\frac{\pi z}{2 a}\right) \sinh \left(\frac{\pi z}{2 a}\right)$

In the $\left(u^{\prime}, v^{\prime}\right)$ the points $A$ and $B$ have the coordinates:

$$
u_{A}^{\prime}=\cos \frac{\pi \alpha}{2}=-u_{B}^{\prime}
$$

With the following conformal transformation (from the $\omega$ ' to the $\omega$-plane):

$$
\omega=\omega^{\prime} \frac{1}{\cos \frac{\pi \alpha}{2}}
$$

the points $A$ and $B$ will move now to the coordinates $(-1,0)$ and $(+1,0)$ in the $(u, v)$ or the $\omega$-plane (Figure $4 d)$. The upper half plane of Figure $4 \mathrm{~d}$ can now be mapped with the inverse transformation of (1) into a half infinite strip with a width $\pi$ as shown in Figure 4e:

$$
\zeta=\xi+j \eta=\arcsin \omega
$$

Why are all these subsequent transformations needed? In the final $\zeta$-plane (Figure $4 \mathrm{e}$ ) we observe a half infinite strip without any cracks. Hence, the electric field and potential distribution can be solved by inspection. Indeed, the current density is just uniform and parallel to the $\eta$ axis. The potential varies linearly with $\eta$. The complex potential in the $\zeta$ plane is then:

$$
W(\zeta)=\frac{2}{\pi}(\varphi+j \psi)=-\mathrm{j} \frac{2}{\pi} \zeta=\frac{2}{\pi}(\eta-j \xi)
$$

$\varphi$ is the potential we are looking for. The factor $2 / \pi$ is just a proportionality constant used for normalization. The complex potential being invariant for conformal transformations, one just has to change the coordinates to obtain the potential in the original track. By using the transformations (1), (2), (3) and (4) listed above, one gets the complex potential in the original $z$-plane, that is, the track with the crack:

$$
W(z)=\frac{2}{\pi}(\varphi(\mathrm{z})+j \psi(\mathrm{z}))=-\mathrm{j} \frac{2}{\pi} \arcsin \frac{\sin \frac{\pi z}{2 a}}{\cos \frac{\pi \alpha}{2}}
$$

The real part of (6) gives us the potential $\varphi(z)=\varphi(x, y)$. For our problem we do not need a detailed potential distribution as a function of $x$ and $y$. We just want to learn the influence of the crack on the electric resistance of the track. Hence, we can limit ourselves to the potential distribution along the $y$ axis:

$$
W(z=j y)=-j \frac{2}{\pi} \arcsin \left[\frac{\sin \frac{\pi y}{2 a}}{\cos \frac{\pi \alpha}{2}}\right]
$$

which is found by putting $x=0$ or $z=j y$ in (7). The real part of (7) gives us the electric potential distribution along the $y$ axis:

$$
\varphi(x=0, y)=\frac{2}{\pi} \ln \left[\frac{\sin \frac{\pi y}{2 a}}{\cos \frac{\pi \alpha}{2}}+\sqrt{\left.1+\frac{\sinh ^{2} \frac{\pi y}{2 a}}{\cos ^{2} \frac{\pi \alpha}{2}}\right]}\right.
$$

A plot of the potential function (8) is shown in Figure 5 for a particular value $\alpha=0.83913$ or $\cos [\pi \alpha / 2]=0.25$ which corresponds to a deep crack in the track. 
For small values of $y(y / a<0.2)$ the potential gradient is much steeper. This is due to the geometric influence of the crack. The supplied electric current has to pass through a narrow slit having a width of only $a(1-\alpha)$ which gives rise to local high electric fields or potential gradients.

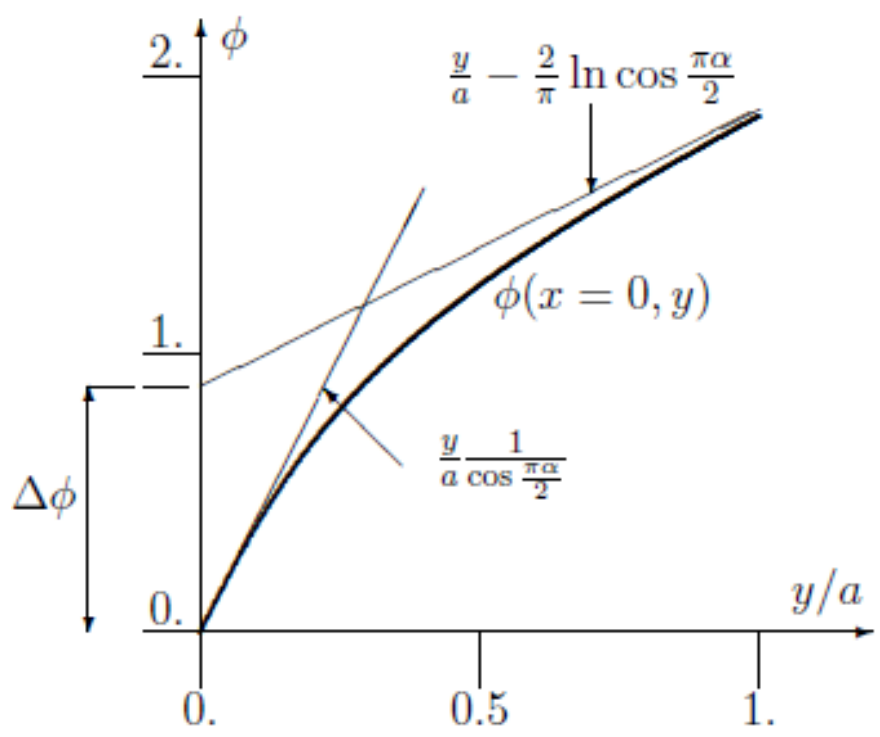

Figure 5. Potential distribution along the $y$-axis or $x=0$

For high values of $y$, the potential function (8) can be approximated by:

$$
\varphi(x=0, y) \approx \frac{\mathrm{y}}{\mathrm{a}}-\frac{2}{\pi} \ln \cos \frac{\pi \alpha}{2}
$$

For small values of $y(y / a<0.2)$ one gets the approximation:

$$
\varphi(x=0, y) \approx \frac{\mathrm{y}}{\mathrm{a}} \frac{1}{\cos \frac{\pi \alpha}{2}}
$$

Both approximations (9) and (10) are also shown in Figure 4.

If there would be no crack in the track (i.e. $\alpha=0$ ) the potential function (8) is simplified to:

$$
\varphi(x=0, y)=\frac{\mathrm{y}}{\mathrm{a}}
$$

The approximation (9) can now be interpreted as the sum of the no crack potential with an offset $\Delta \varphi$ (Figure 4) given by:

$$
\Delta \varphi=-\frac{2}{\pi} \ln \cos \frac{\pi \alpha}{2}
$$

As the entire calculation outlined above was carried out under the assumption of a constant electric current flowing through the track, the extra electric resistance $\Delta R$ due the crack is also proportional to $\Delta \varphi$. A graphical representation of $\Delta \varphi$ is shown in Figure 6.

For small values of $\alpha$ the function (12) can be approximated by:

$\Delta R \propto \Delta \varphi=-\frac{2}{\pi} \ln \cos \frac{\pi \alpha}{2}=-\frac{2}{\pi} \ln \left(1-\frac{\alpha^{2} \pi^{2}}{8}\right) \approx \frac{\alpha^{2} \pi}{4}$ which indicates clearly a parabolic behaviour. It means that in the beginning when the crack starts growing, the resistance will increase parabolically with the depth of the crack. The approximation (13) has also been drawn in Figure 5, which is just a straight line due to the quadratic scale used for the horizontal axis. It turns out that the approximation (13) is quite good for values $\alpha<0.5$.

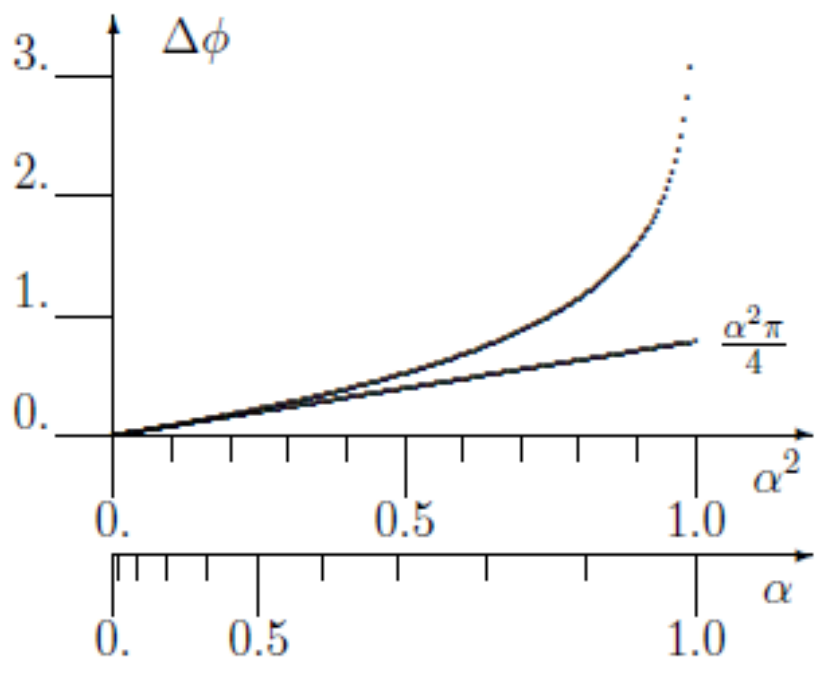

Figure 6. Potential offset $\Delta \varphi$ as a function of $\alpha$

The quadratic relation (13) we found is the reason why a trendline $\left(\propto N^{2}\right)$ has been drawn in Figures 2 and 3 . First we assume for a while that just a single crack exist in each sample. Second, it is assumed that the crack length grows with the same amount during each cleaning cycle. Then it will be found that the change of the electric resistance $\Delta R$ will be proportional to $N^{2}$ as long as the number $N$ is not too high.

\section{$\underline{3.2}$ Numerical modelling}

In the theoretical analysis, several assumptions have been made: (1) the sample should have the shape of a long track, (2) the crack is perpendicular to the boundary, (3) only one crack is built up, and (4) the crack grows linearly with the number of cleaning cycles.

For the experimental measurements, square shaped resistors were used. Consequently, for the simulation part or this research square shaped resistors were used as well. A sample is then replaced by a square shaped network of resistors ordered in a regular array as shown in Figure $7 a$.

To avoid confusion with the individual resistors $R$ in Figure 7 we call the sample as the global resistance. Two contacts at potentials $\varphi=1 \quad V$ and $\varphi=0 \quad V$ are provided. By solving this electric network numerically, one obtains the supplied current $I_{0}$. The global resistance, we are interested in, is then given by $R_{0}=1 \mathrm{~V} / \mathrm{I}_{0}$.

It should be remarked that solving the resistor network of Figure $7 a$ is mathematically equivalent to the numerical solution of the Laplace equation using the finite difference approximation [18-19]. To fulfill the zero potential gradient condition at the 
vertical free boundaries, resistors with a double value $2 R$ were introduced there. For the numerical solution, an interactive method, known as the relaxation technique, has been used [19].

Simulations have been carried out for conducting sheets, which have been divided into $100 \times 100$ cells. In Figure 7 , only $3 \times 3$ cells were drawn for the sake of clarity. A crack is simulated by removing a single resistor from the network as shown in Figure $7 \mathrm{~b}$. This resistor was chosen arbitrarily by using a random generator in the simulation programme. The new network, with one resistor removed, is then solved numerically as well, giving rise to a new (and usually slightly higher) current $I_{1}$ and a new global resistance value $R_{1}=1 \mathrm{~V} / \mathrm{I}_{1}$. Obviously $R_{1} \geq R_{0}$. Then a second, also randomly selected resistor is removed (Figure 7c). This gives rise to a new global resistance value given by $R_{2}=1 \mathrm{~V} / I_{2}\left(R_{2} \geq R_{1} \geq R_{0}\right)$. This procedure is repeated till the number of removed resistors is that high that no electric conduction is possible.

The results obtained with a $100 \times 100$ cell are shown in Figure 8 .

For this simulation a value $R=1 \Omega$ was used. One can easily verify that the global resistance is also $1 \Omega$ at the beginning, that is, before any resistor has been removed (Figure 7a). One will remark that the global resistance increases dramatically when the number of removed resistors $N_{R}>6.000$. For $N_{R}>10.500$ no electric conduction was possible. For a $100 \times 100$ cell, we have in total 20.000 resistors in the network. The same problem was simulated many times. All results were similar except for the value of $N_{R}$ where the conduction stops. In our simulation, conduction stops when there is no longer any conducting path between the two electrodes.

Because the theoretical section revealed a clear quadratic behaviour (see eq. (13), the results of Figure 8 have been plotted again in Figure 9 but using a quadratic scale on the horizontal axis. For values $N_{R}<6000$, the plot of Figure 9 can be very well fitted to a straight line, in full agreement with the theoretical analysis.

The trendline shown in Figure 9 is given by the equation:

$$
R-R_{0}=4.4852 \cdot 10^{-8} N_{R}^{2}
$$

where $R_{0}=1 \Omega$ as already mentioned. It must be emphasised here that similar results have been obtained for many runs of the simulation programme. The slope of the trendline was always around the value $4.485210^{-8}$ with a maximum deviation of no more than $\pm 5 \%$. Several simulations have also been carried out for a $50 \times 50$ cell. All results were still in full agreement with the previous simulations.
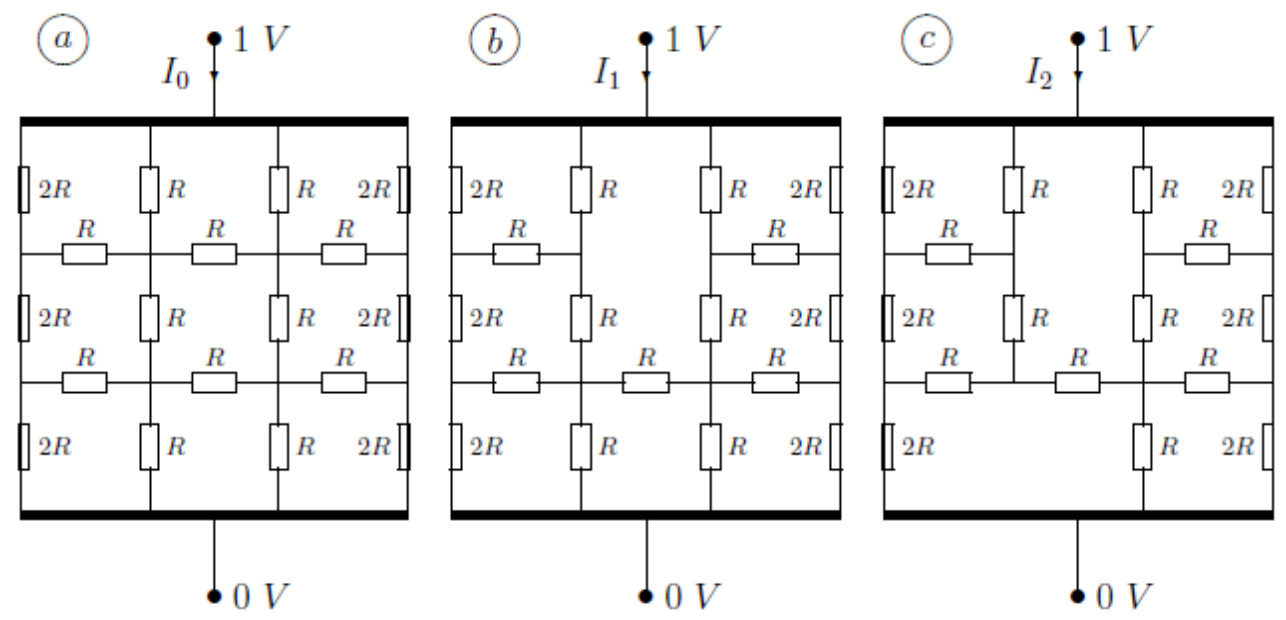

Figure 7. Network of resistors in a regular array to simulate the global resistance of a sheet. Cracks are simulated by removal of a single resistor..

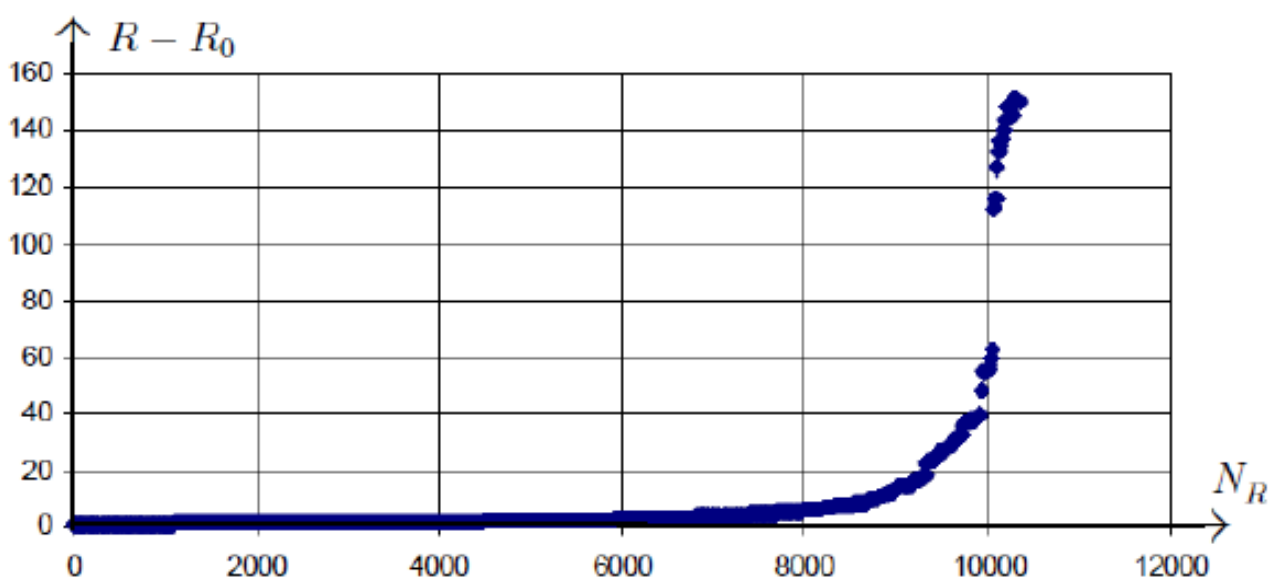

Figure 8. Numerical simulated ageing behaviour showing the global resistance change versus the number of removed resistors $N_{R}$ 


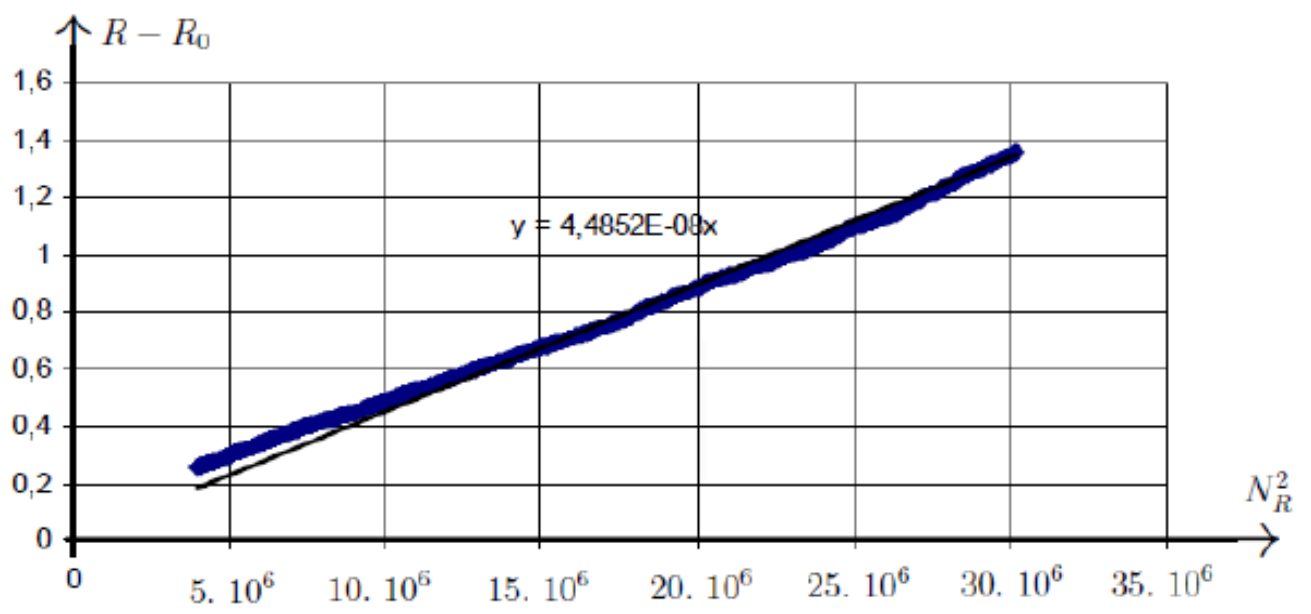

Figure 9. Numerical simulated ageing behaviour showing the global resistance change versus the square of the number of removed resistors $N_{R}^{2}$

Needless to say that one dry cleaning cycle can correspond to the removal of one or more resistors. It means that the number $N$ of dry cleaning cycles is then a multiple of $N R$.

\section{Conclusion}

Several textile samples were made electroconductive through screen printing electrically conducting silver-based inks. Any mechanical deformation of the sample may cause a crack or microcrack in the screen printed layer, giving rise to an increase in the total electric resistance.

For most samples it was observed that the resistance increased proportionally with the square of the number of dry cleaning cycles. Only the sample deposited on nonflexible substrates showed a much smaller increase of the electric resistance.

The increase of the electric resistance caused by a cut in a long track was evaluated using conformal mapping techniques. It was found that the resistance increases with the square of the cut's length. If one assumes that each dry cleaning cycle increases the length of the cut by the same amount, this analysis explains the square law behaviour observed experimentally.

A dry cleaning cycle was simulated by the removal of one or more of these resistors. It was again found that the total resistance increases with the square of the number of removed resistors. Hence, the numerical simulation also confirmed the experimental observations.

\section{Acknowledgement}

I. Kazani wants to thanks the EU for the financial support for her stay as a Phd student at Ghent University within the framework of the Basileus programme.

R. Klepacko wants to thank the EU for the financial support for his stay at Ghent University within the framework of the students exchange programme Eramus.

\section{References}

[1] MeoliD., May-PlumleeT. 2002. Interactive electronic textile development: A review of technologies» Journal of Textile and Apparel Technology and Management. vol.2, p.1-12.

[2] Merilampi S., Laine-Ma T., Ruuskanen P. 2009. The characterisation of electrically conductive silver ink patterns on flexible substrates. Microelectronics Reliability. vol.49, p.782-790.

[3] Yang Y.L., Chuang M.C., Lou S.L., Wang J. 2010. Thickfilm textile-based amperometric sensors and biosensors. Royal Society of Chemistry. vol.135, p.1230-1234.

[4] Kim Y., Kim H., Yoo H.J. 2010. Electrical characterisation of screen - printed circuits on the fabric. IEEE Transactions on Advanced Packaging. vol. 33, p. 196-205.

[5 Karaguze IB. Using conductive inks and nonwoven textiles for wearable computing.http://www.ntcresearch. org/projectapp/project_pages/F04-NS17/pdf_files/ Karaguzel\%20-\%20TIWC\%20SPRG\%20Paper.pdf/. NTC Project: F04-NS17. - North Carolina

[6] Karaguzel B., Merritt CR., Kang T., Wilson J.M, Nagle HT, Grant E, Polurdeyhimi B. 2009. Flexible, durable printed electrical circuits. The Journal of the Textile Institute. vol.100, p.1-9.

[7] Merritt C. R., Karaguzel B., Kang T-H., Wilson J. M., Franzon P.D.,. Nagle H. T, Poudeyhimi B., Grant E. 2005. Electrical characterization of Transmission Lines on Nonwoven Textile substrates. Material Research Society. vol. 870, p. 87-95.

[8] Hertleer C., Van Langenhove L., Rogier H. 2008. Printed Textile Antennas for Off-body Communication. Advances in Science and Technology. vol.60, p.64-66.

[9] Scarpello M. L., Kazanil., Hertleer C., Rogier H., Ginste D. V. 2012. Stability and efficiency of screen-printed wearable and washable antennas. IEEE Antennas and Wireless Propagation Letters. vol.11, p.838 - 841.

[10] Kazani I., Hertleer C., De Mey G., Guxho G., Van Langenhove L. 2013. Dry cleaning of electroconductive layers screen printed on flexible substrates. Textile Research Journal. vol. 83, p. 1541-1548.

[11] Varnaité S., Katunskis J. 2009. Influence of washing on the electric charge decay of fabrics with conductive yarns. FIBRES \& TEXTILES in Eastern Europe 5 (76), vol.17, p.69-75. 
[12] Varnaité S., Vitkauskas A., Abraitiené A., Rubžiené V., Valiené $V$. 2008. The features of electric charge decay in the Polyester Fabric containing metal fibres. Materials Science. vol.14, p.157-161.

[13] Van Der Pauw L. J. 1958. A method of measuring specific resistivity and Hall effect of discs of arbitrary shape. Philips Research Reports. vol.13, p.1-9.

[14] Banaszczyk J., De Mey G., Schwarz A., Van Langenhove L. 2010. The Van Der Pauw method for sheet resistance measurements of polypyrole coated para aramide woven fabrics» Journal of Applied Polymer Science, vol.117, p.2553-2558.
[15] Kazani I., De Mey G., Hertleer C., Banaszczyk J., Schwarz A., Guxho G., Van Langenhove. L. 2011. Van der Pauw method for measuring resistivities of anisotropic layers printed on textile substrates. Textile Research Journal. vol.81, p.2117-2124.

[16] Churchill R. 1960. Complex variables and applications. Mc Graw Hill, NY, p.191-195.

[17] Kober H. 1957. Dictionary of conformal representations. Dover, NY, p.95-96.

[18] Abramowitz M. and Stegunl. 1970. Handbook of mathematical functions. Dover, New York, p.885.

[19] Cengel Y. 2003. Heat transfer. Mc Graw Hill, Boston, p.281-291. 